\title{
Constructing Restricted Patterson Measures for Geometrically Infinite Kleinian Groups
}

\author{
Kurt FALK ${ }^{1)}$ \\ Mathematical Institute, University of Bern, Sidlerstrasse 5, CH-3012 Bern, Switzerland \\ E-mail: kurt.falk@math-stat.unibe.ch \\ Bernd O. STRATMANN \\ Mathematical Institute, University of St Andrews, North Haugh, \\ St Andrews KY16 9SS, Scotland, UK \\ E-mail:bos@st-and.ac.uk
}

\begin{abstract}
In this paper, we study exhaustions, referred to as $\rho$-restrictions, of arbitrary nonelementary Kleinian groups with at most finitely many bounded parabolic elements. Special emphasis is put on the geometrically infinite case, where we obtain that the limit set of each of these Kleinian groups contains an infinite family of closed subsets, referred to as $\rho$-restricted limit sets, such that there is a Poincaré series and hence an exponent of convergence $\delta_{\rho}$, canonically associated with every element in this family. Generalizing concepts which are well known in the geometrically finite case, we then introduce the notion of $\rho$-restricted Patterson measure, and show that these measures are non-atomic, $\delta_{\rho}$-harmonic, $\delta_{\rho}$-subconformal on special sets and $\delta_{\rho}$-conformal on very special sets. Furthermore, we obtain the results that each $\rho$-restriction of our Kleinian group is of $\delta_{\rho}$-divergence type and that the Hausdorff dimension of the $\rho$-restricted limit set is equal to $\delta_{\rho}$.
\end{abstract}

Keywords Kleinian group, Patterson measure, Hausdorff dimension MR(2000) Subject Classification 30F40; 37F35

\section{Introduction}

In this paper we consider arbitrary non-elementary Kleinian groups with at most finitely many bounded parabolic elements. Special emphasis will be put on geometrically infinite groups (that is, groups which have fundamental domains with infinitely many faces), although strictly speaking our analysis also applies to the geometrically finite situation where it resembles the well-known results from the Patterson-Sullivan theory for geometrically finite Kleinian groups.

Recall that every arbitrary Kleinian group admits the construction of the classical Patterson measure, a measure which is canonically obtained from the orbit structure of the group action, and which is always supported on the limit set of the group. For geometrically finite groups this measure is well understood and has proven to be an extremely fruitful tool in the studies of Kleinian groups under various aspects, such as, for instance, under aspects of group cohomology, or of spectral analysis on hyperbolic manifolds, or also of fractal geometry on limit sets. However, for the class of geometrically infinite Kleinian groups, the situation appears to be completely different and less satisfying. For instance, for the so-called $\delta$-convergence type Kleinian groups (that is, groups whose associated Poincaré series converge at their abscissa of convergence, and which are necessarily geometrically infinite), Sullivan has shown that the

Received March 18, 2004, Accepted October 25, 2004

1) Research supported by the Schweizer Nationalfonds No. 20-61379.00 and European Project TMR "Geometric Analysis" ACR-OFES No. UE 00.0349 
geodesic flow on the associated hyperbolic manifolds is no longer ergodic with respect to the Liouville-Patterson measure, a property which is equivalent to the fact that the Patterson measure vanishes on the set of so-called radial limit points $([1,2])$. In other words, for this class of hyperbolic manifolds the Patterson measure appears to be no longer a useful concept for studying recurrent geodesic dynamics. For this reason it is usually rather difficult, not to say impossible, to transfer the successful techniques, which have been elaborated in connection with this measure in the geometrically finite case, to the studies of geometrically infinite groups by means of their Patterson measure.

In this paper we show how to avoid these difficulties by setting up a new type of Patterson measure, referred to as the restricted Patterson measure. These measures are specially designed to allow investigations of recurrent geodesic dynamics of the geometrically finite type within geometrically infinite hyperbolic manifolds. The idea is to consider finite volume regions of the convex core of the manifold, and to associate with each of these an orbital measure which allows us to quantify their internal long-range geodesic dynamics. More precisely, within the manifold we consider the family of balls of radius $\rho$ centred at some fixed base point, where $\rho$ is assumed to be sufficiently large such that the balls engulf the regions where the intersections of the convex core with the bounded cusps meet the thick part of the manifold. The finite volume regions, mentioned above, are then given by the so-called restricted cores, that is, the intersection of the convex core with the union of the bounded cusps and the $\rho$-ball. By embedding these restricted cores into the universal covering of the manifold and introducing a coarse geometric way of identifying asymptotic geodesic behaviour within them, we obtain certain subsets $G_{\rho}$ of the underlying Kleinian group $G$, which we refer to as $\rho$-restrictions. Now, each of these $\rho$-restrictions acts on hyperbolic space and hence, by considering the $G_{\rho}$-orbit of some point in hyperbolic space, it gives rise to a limit set $L\left(G_{\rho}\right)$, referred to as the $\rho$-restricted limit set. Clearly, each $L\left(G_{\rho}\right)$ is, by construction, a closed subset of the limit set of $G$. Also, with each $\rho$-restriction, we can associate a Poincaré series $\mathscr{P}_{\rho}(s)$ and hence an exponent of convergence $\delta_{\rho}$, which then allows us to mimic the construction of the classical Patterson measure within this 'restricted setting'. In this way we derive, for any arbitrary observation points $x$ in the hyperbolic space, our so-called $\rho$-restricted Patterson measure $\mu_{\rho}^{x}$ with support equal to $L\left(G_{\rho}\right)$. The paper continues by giving a geometric analysis of the class of $\rho$-restricted Patterson measures. The main results of this analysis are the following (Theorem 3.3):

- The measure $\mu_{\rho}^{x}$ is non-atomic.

- $G_{\rho}$ is of $\delta_{\rho}$-divergence type, meaning that $\mathscr{P}_{\rho}(s)$ diverges for $s=\delta_{\rho}$.

- The Hausdorff dimension of $L\left(G_{\rho}\right)$ is equal to $\delta_{\rho}$.

Part of the proof of this theorem is to show that the Poincaré exponent $\delta(G)$ of $G$ and the exponents $\delta_{\rho}$ of the $\rho$-restrictions are related as follows. We remark that this result may also be of independent interest in the theory of dynamical systems.

- $\lim _{\rho \rightarrow \infty} \delta_{\rho}=\delta(G)$. (Proposition 3.1)

Subsequently, we also derive the following two results, which mark the similarities between the classical and the $\rho$-restricted Patterson measures. Here $\delta_{\rho}$-harmonic refers to the fact that the Radon-Nikodym derivative of $\mu_{\rho}^{x}$ with respect to $\mu_{\rho}^{0}$ can be expressed in terms of the $\delta_{\rho}$-th power of the Poisson kernel. Note that with this notation the classical Patterson measure is in fact $\delta(G)$-harmonic. Also, note that as a consequence of the $\delta_{\rho}$-harmonicity we have that the measure class of $\mu_{\rho}^{x}$ is invariant under changes of the observation point $x$. We refer to the end of Section 3 for a more detailed definition of $\delta_{\rho}$-harmonic and for the meaning of ' $\delta_{\rho}$-subconformal on special sets' and ' $\delta_{\rho}$-conformal on very special sets'.

- The measure $\mu_{\rho}^{x}$ is $\delta_{\rho}$-harmonic. (Lemma 3.4)

- The measure $\mu_{\rho}^{x}$ is $\delta_{\rho}$-subconformal on special sets, and it is $\delta_{\rho}$-conformal on very special sets. (Lemma 3.5)

We remark that Kleinian groups of $\delta(G)$-convergence type provide a particularly interesting 
class of examples to which our analysis applies. Clearly, since $\rho$-restrictions are of $\delta_{\rho}$-divergence type, for these groups we have that $\delta_{\rho}$ is always strictly less than $\delta(G)$. Note that this class of examples includes all geometrically infinite, finitely generated Kleinian groups acting in hyperbolic 3 -space which have the property that the area of their limit sets vanishes. (This follows since in this case $\delta(G)=2([3,4])$, and hence 2-divergence type would imply that the radial limit set is of full Patterson measure ([5]), contradicting the fact that the area of the limit set vanishes. Also note that Sullivan has shown that these groups have only finitely many non-equivalent parabolic elements $([6]))$. On the other hand, for geometrically finite Kleinian groups, our analysis is trivial in the sense that it leads to well-known results from the PattersonSullivan theory. (This follows since, in this case, the $\rho$-restricted cores which we consider are always equal to the convex core of the manifold, and therefore $\delta_{\rho}$ is always equal to $\delta(G)$.) These observations clearly illustrate in which way the constructions in this paper generalize the classical Patterson measure for geometrically finite groups.

The paper is organised as follows. In Section 2 we first introduce the basic notions in connection with our 'concept of restrictions', and then discuss a few technical observations which will be required later. Section 3 starts with the construction of the $\rho$-restricted Patterson measures. This is followed by a finer analysis of these measures, which then leads to the main results of this paper. Furthermore, the appendix contains a detailed description of 'the method of a slowly varying function', which was first used by Patterson in [7] to construct orbital measures on limit sets of Fuchsian groups (and which is referred to by Sullivan as 'nifty' in his generalizations of the Patterson measure to Kleinian groups $([8]))$. We have included this description, since our construction is a slightly modified version of Patterson's original construction, and also mainly because the literature seems not to contain a description of this method in all its details (and hence we hope that the reader may find it helpful to see such a detailed discussion).

Finally, we remark that this paper was originally inspired by the generalizations of the notion of conformal measure to Julia sets of rational maps containing critical points by Denker and Urbański (see e.g. $[9,10,11]$ ). However, it seems that their construction, which uses ergodic theory and, in particular, the thermodynamical formalism, is only vaguely connected to our construction in this paper, which is more canonical in the sense that it is purely geometric.

Throughout, we use the following conventions to describe the relationship between two positive real numbers $a$ and $b$. We write $a \asymp b$ to mean that the ratio of $a$ and $b$ is uniformly bounded away from 0 and infinity, and we write $a \ll b$ if $a / b$ is uniformly bounded from above. Furthermore, if $\exp (a) \asymp \exp (b)$, then we write $a \asymp+b$.

\section{Restricted Limit Sets and Their Geometry}

We assume that the reader is familiar with the basic theory of Kleinian groups (see e.g. [12, 13]). Throughout, let $G$ be a non-elementary Kleinian group with a at most, finite set $P=$ $\left\{p_{1}, \ldots, p_{k}\right\}$ of non-equivalent bounded standard parabolic fixed points (where $P$ is allowed to be the empty set). It is well known that, with each $p \in P$, we can associate a horoball $\mathscr{H}_{p}$ intersecting a fundamental domain at the origin such that the $G$-orbit $G(\mathscr{H}):=\left\{g\left(\mathscr{H}_{p}\right): g \in\right.$ $G, p \in P\}$ represents a packing of hyperbolic space $\mathbb{D}^{N+1}$ by mutually pairwise disjoint balls tangent to the boundary $S^{N}$ of $\mathbb{D}^{N+1}$. Let $\mathscr{C}=\mathscr{C}(G)$ denote the convex hull within $\mathbb{D}^{N+1}$ of the limit set $L(G)$ of $G$, and choose $0 \in \mathbb{D}^{N+1}$ as a fixed reference point. Fix $\rho_{0}$ such that the geodesics between different elements of $P$ are contained in $B_{\rho_{0}}(0) \cup \bigcup_{p \in P} \mathscr{H}_{p}$, and such that, for the horospherical boundaries $H_{p}$ of the horoballs $\mathscr{H}_{p}$, we have that $\bigcup_{g \in G} g\left(B_{\rho_{0}}(0)\right) \supset$ $\mathscr{C} \cap \bigcup_{p \in P} \bigcup_{g \in G} g\left(H_{p}\right)$. (Here, $B_{r}(z)$ refers to the open hyperbolic ball centred at $z$ of radius $r$.) For $\rho>\rho_{0}$, we define the $\rho$-restricted convex core of $G$ by

$$
\mathscr{C}_{\rho}:=\mathscr{C} \cap\left(G(\mathscr{H}) \cup \bigcup_{g \in G} g\left(B_{\rho}(0)\right)\right) .
$$


Also, we introduce the notion of the core shade $S_{\rho}(x, y)$, which is given for $x, y \in \mathbb{D}^{N+1}$ such that $B_{\rho}(x) \cap B_{\rho}(y)=\emptyset$, by

$$
S_{\rho}(x, y):=\left\{z \in \mathbb{D}^{N+1} \backslash B_{\rho}(y): s_{w, z} \cap B_{\rho}(y) \neq \emptyset \text { for all } w \in B_{\rho}(x)\right\} .
$$

Here, $s_{x, y}$ refers to the geodesic segment between $x$ and $y$. Note that a core shade is by definition an open subset of $\mathbb{D}^{N+1}$. Furthermore, if the boundary at infinity $\partial E$ of a set $E \subset \mathbb{D}^{N+1}$ is defined as the Euclidean interior in $S^{N}$ of $\bar{E} \backslash(E \cup \operatorname{cl}(E))$, where $\operatorname{cl}(\cdot)$ refers to the closure in $\mathbb{D}^{N+1}$, and - to the closure in $\mathbb{R}^{N+1}$, then we have, for the boundary at infinity of $S_{\rho}(x, y)$, that

$$
\partial S_{\rho}(x, y)=\bigcap_{w \in B_{\rho}(x)} \Pi_{w}\left(B_{\rho}(y)\right) .
$$

Here, $\Pi_{w}$ refers to the shadow projection based at $w \in \mathbb{D}^{N+1}$, which is given for $E \subset \mathbb{D}^{N+1}$ by $\Pi_{w}(E):=\left\{\xi \in S^{N}: s_{w, \xi} \cap E \neq \emptyset\right\}$.

Finally, we define the $\rho$-restricted core shade

$$
\widehat{S}_{\rho}(x, y):=\left(\mathbb{D}^{N+1} \backslash \mathscr{U}_{\rho}\left(\mathbb{D}^{N+1} \backslash S_{\rho}(x, y)\right)\right) \cup \overline{\partial S_{\rho}(x, y)},
$$

where $\mathscr{U}_{\rho}(\cdot)$ refers to the open hyperbolic $\rho$-neighbourhood of a subset of $\mathbb{D}^{N+1}$. Note that $\widehat{S}_{\rho}(x, y)$ is, by construction, a closed subset of $\mathbb{D}^{N+1}$.

The following definition introduces the types of limit sets which will be important throughout the paper:

Definition 2.1 Let $\rho>\rho_{0}$ and $g \in G$ be given. A geodesic $l$ is called $(\rho, g)$-visible if and only if it is fully contained in $\mathscr{C}_{\rho}$ and intersects $B_{\rho}(g(0))$. The $(\rho, g)$-restriction $G_{\rho}^{g}$ is then given by

$$
G_{\rho}^{g}:=\left\{h \in G: l \cap B_{\rho}(h(0)) \neq \emptyset \text { for some }(\rho, g) \text {-visible } l\right\},
$$

and the $(\rho, g)$-restricted limit set $L\left(G_{\rho}^{g}\right)$ of $G$ is the derived set of $G_{\rho}^{g}(0)$, which is defined by

$$
L\left(G_{\rho}^{g}\right):=\overline{G_{\rho}^{g}(0)} \backslash G_{\rho}^{g}(0) .
$$

Also, we introduce the following two subsets of $L\left(G_{\rho}^{g}\right)$ :

- The set of $\rho$-radial limit points of $G_{\rho}^{g}$ will be denoted by $L_{r}\left(G_{\rho}^{g}\right)$. Here $\xi$ is called $\rho$-radial if there exists a geodesic $l$ in $\mathrm{cl}\left(\mathscr{C}_{\rho}\right)$ with endpoint $\xi$ such that $l$ intersects $\mathrm{cl}\left(B_{\rho}(g(0))\right)$, and such that each ray $r \subset l$ towards $\xi$ intersects $\operatorname{cl}\left(B_{\rho}(h(0))\right)$ for infinitely many $h \in G_{\rho}^{g}$.

- The set of bounded parabolic fixed points of $G$ which are contained in $L\left(G_{\rho}^{g}\right)$ will be denoted by $L_{p}\left(G_{\rho}^{g}\right)$.

For ease of notation, we let $G_{\rho}:=G_{\rho}^{\{\text {id. }\}}$, and if a geodesic $l$ is $(\rho,\{$ id. $\})$-visible then we shall refer to it as $\rho$-visible.

We now collect a few observations which will turn out to be helpful in our measuretheoretical analysis of the following section. We shall see first that $L\left(G_{\rho}^{g}\right)$ can be written as the disjoint union of $L_{p}\left(G_{\rho}^{g}\right)$ and $L_{r}\left(G_{\rho}^{g}\right)$. This observation represents an analog of the well-known corresponding result of Beardon and Maskit in the geometrically finite case (cf. [14]).

Proposition 2.2 For $\rho>\rho_{0}$ and $g \in G$ the following hold:

(i) For each $\xi \in L\left(G_{\rho}^{g}\right)$ there exists a geodesic ending at $\xi$ which is contained in $\mathrm{cl}\left(\mathscr{C}_{\rho}\right)$ and has a non-empty intersection with $\mathrm{cl}\left(B_{\rho}(g(0))\right)$.

(ii) $L\left(G_{\rho}^{g}\right)=L_{r}\left(G_{\rho}^{g}\right) \cup L_{p}\left(G_{\rho}^{g}\right)$.

Proof We give the proof for $g=\{$ id. $\}$ and remark that the general case follows exactly in the same way. In order to prove the statement in (i), let $\xi \in L\left(G_{\rho}\right)$ be given. Then there exists a sequence $\left(g_{n}\right)$ in $G_{\rho}$ such that $g_{n}(0)$ tends to $\xi$ in the Euclidean metric. Let $l_{n}$ denote the $\rho$-visible geodesic associated with $g_{n}$ by the definition of $G_{\rho}$, and let $\xi_{n}$ and $\eta_{n}$ denote its endpoints. Without loss of generality we can assume that $\xi_{n}$ tends to $\xi$. It follows that $\left(l_{n}\right)$ accumulates at some geodesic $l$ which terminates at $\xi$. By passing to a subsequence if necessary, we can assume without loss of generality that $l_{n}$ converges to $l$. Since $l_{n}$ is contained in $\mathscr{C}_{\rho}$ for all $n$, and since $\mathscr{C}_{\rho}$ is open, it follows that $l$ is contained in $\operatorname{cl}\left(\mathscr{C}_{\rho}\right)$. Furthermore, each $l_{n}$ has a non-empty intersection with $B_{\rho}(0)$, which implies that $l$ intersects $\operatorname{cl}\left(B_{\rho}(0)\right)$. 
In order to prove the statement in (ii), again let $\xi \in L\left(G_{\rho}\right)$ and let $l$ be the associated geodesic, which we obtained in the proof of (i) above. In this situation a ray towards $\xi$ which is contained in $l$ either intersects $\operatorname{cl}\left(B_{\rho}(g(0))\right)$ for infinitely many $g \in G_{\rho}$, or is eventually contained in $h\left(\mathscr{H}_{p}\right)$, for some $h \in G$ and $p \in P$. In the first case it follows that $\xi \in L_{r}\left(G_{\rho}\right)$. In the second case we conclude that $\xi=h(p) \in L_{p}\left(G_{\rho}\right)$.

For the remaining part of this section we restrict the discussion to the ( $\rho,\{$ id. $\})$-restriction $G_{\rho}$ of $G$. To further clarify the significance of a radial limit point, we introduce the following concept of a $\rho$-trace at a point in $L_{r}\left(G_{\rho}\right)$ :

Definition 2.3 For $\rho>\rho_{0}$ and $\xi \in L_{r}\left(G_{\rho}\right)$, the $\rho$-trace at $\xi$ consists of the optimal sequence $\left(g_{m}\right)$ of elements $g_{m} \in G_{\rho}$ such that $\xi \in \partial S_{\rho}\left(0, g_{m}(0)\right)$ for all $m$ and such that the Euclidean diameter of $\partial S_{\rho}\left(0, g_{m}(0)\right)$ tends to 0 monotonically, for $m$ tending to infinity.

Lemma 2.4 There exists a $\rho$-trace at each $\xi \in L_{r}\left(G_{\rho}\right)$.

Proof Let $\xi \in L_{r}\left(G_{\rho}\right)$ be given. Then there exist a geodesic $l_{\xi}$ in $\operatorname{cl}\left(\mathscr{C}_{\rho}\right)$ ending at $\xi$, and an optimal sequence $\left(g_{m}\right)$ in $G_{\rho}$ with $g_{1}=\{$ id. $\}$ such that $l_{\xi}$ has a non-empty intersection with $\operatorname{cl}\left(B_{\rho}\left(g_{m}(0)\right)\right)$, for all $m$. With $e_{m}$ referring to the hyperbolic distance between $g_{m}(0)$ and $l_{\xi}$, we clearly have that $\liminf e_{m} \leq \rho$. We consider the cases $\liminf e_{m}<\rho$ and $\liminf e_{m}=\rho$ separately.

For liminf $e_{m}<\rho$ we have that there exist $\varepsilon>0$ and a ray $r_{\xi}$ towards $\xi$ with initial point somewhere in $B_{\rho}(0)$, such that $r_{\xi}$ intersects $B_{\rho-\varepsilon}\left(g_{m}(0)\right)$ for all $m$ sufficiently large. If we assume that there is no $\rho$-trace at $\xi$, then there exists a ray $r_{\xi}^{*}$ towards $\xi$ with an initial point somewhere in $B_{\rho}(0)$, such that $r_{\xi}^{*}$ has an empty intersection with $B_{\rho}\left(g_{m}(0)\right)$ for all $m$ sufficiently large. Combining these two observations, it follows that there exists a nested sequence $\left(H_{m}(\xi)\right)$ of horospheres based at $\xi$ such that for $x_{m}:=H_{m}(\xi) \cap r_{\xi}$ and $x_{m}^{*}:=H_{m}(\xi) \cap r_{\xi}^{*}$ we have that $d\left(x_{m}, x_{m}^{*}\right) \geq \varepsilon$ for all $m$ sufficiently large. On the other hand, recall that $r_{\xi}$ and $r_{\xi}^{*}$ are asymptotic which gives that $\lim _{m \rightarrow \infty} d\left(x_{m}, x_{m}^{*}\right)=0$, and hence leads to a contradiction.

If $\lim \inf e_{m}=\rho$, then we in fact have that $\lim e_{m}=\rho$, and we now show that this case cannot occur. Let $r_{\xi}^{\prime}$ denote some ray towards $\xi$ which starts in $\operatorname{cl}\left(B_{\rho}(0)\right)$ and which is contained in $l_{\xi}$. Let $\sigma_{m}:=r_{\xi}^{\prime} \cap \operatorname{cl}\left(B_{\rho}\left(g_{m}(0)\right)\right)$, and note that the hyperbolic length of $\sigma_{m}$ tends to 0 for $m$ tending to infinity. Note that eventually $\sigma_{m}$ has to lie outside $G(\mathscr{H})$, since otherwise $r_{\xi}^{\prime}$ would leave $G(\mathscr{H})$ infinitely often and hence would intersect $B_{c}(h(0))$ for infinitely many $h \in G_{\rho}$, for some constant $c>0$ which depends only on the distance of 0 to $\mathscr{H}$. This clearly contradicts the fact that the hyperbolic length of $\sigma_{m}$ tends to 0 . Now, consider geodesic segments of some fixed hyperbolic length which are contained in $r_{\xi}^{\prime}$ and which are sufficiently far away from the origin. Again since the hyperbolic length of $\sigma_{m}$ tends to 0 , we have that the number of balls $\operatorname{cl}\left(B_{\rho}\left(g_{m}(0)\right)\right)$ required to cover such a segment increases if the distance of the segment to the origin grows. This clearly contradicts the fact that $G_{\rho}$ acts discontinuously on $\mathbb{D}^{N+1}$, and hence the lemma follows.

Finally, we now outline a few algebraic properties of $G_{\rho}$. First note that since the composition of two elements of $G_{\rho}$ is not necessarily again an element of $G_{\rho}$, it follows that in general we do not have that $G_{\rho}$ is a group. Nevertheless, as we shall see now, $G_{\rho}$ has certain weaker properties of invariance under compositions.

Lemma 2.5 With the notation above, the following hold:

(i) $\{$ id. $\} \in G_{\rho}$;

(ii) $g \in G_{\rho}$ if and only if $g^{-1} \in G_{\rho}$;

(iii) If $g \in G$ and $h \in G_{\rho}$ are such that $B_{\rho}(g(0))$ does not intersect $B_{\rho}(0)$, and $B_{\rho}(h(0))$ is contained in $S_{\rho}(0, g(0))$, then $g, g^{-1} h \in G_{\rho}$.

Proof The statements in (i), (ii) and the conclusion in (iii) that $g \in G_{\rho}$ are immediate consequences of the definition of $G_{\rho}$. In order to prove the remaining assertion, let $l$ be a $\rho$-visible geodesic which intersects $B_{\rho}(h(0))$. Since $B_{\rho}(h(0))$ is a subset of $S_{\rho}(0, g(0))$, we have by defini- 
tion of $S_{\rho}(0, g(0))$ that $l$ has a non-empty intersection with $B_{\rho}(g(0))$. Hence, $g^{-1}(l)$ is contained in $\mathscr{C}_{\rho}$ and intersects each of the balls $B_{\rho}\left(g^{-1}(0)\right), B_{\rho}(0)$ and $B_{\rho}\left(g^{-1} h(0)\right)$. This implies that $g^{-1} h \in G_{\rho}$.

As an immediate consequence of the preceeding lemma we have the following corollary which will be required in Section 3:

Corollary 2.6 For $g \in G_{\rho}$ such that $B_{\rho}(g(0))$ does not intersect $B_{\rho}(0)$, the following hold:

(i) $\left\{g h: h \in G_{\rho}, g h(0) \in \widehat{S}_{\rho}(g(0), 0) \subset\left\{k \in G_{\rho}: k(0) \in \widehat{S}_{\rho}(g(0), 0)\right\}\right.$;

(ii) $\left\{k \in G_{\rho}^{g}: \begin{array}{l}\text { there exists a geodesic } l \subset \operatorname{cl}\left(\mathscr{C}_{\rho}\right) \cap \operatorname{cl}\left(S_{\rho}(g(0), 0)\right) \\ \text { intersecting } \operatorname{cl}\left(B_{\rho}(0)\right), \operatorname{cl}\left(B_{\rho}(g(0))\right) \text { and } \operatorname{cl}\left(B_{\rho}(k(0))\right)\end{array}\right\}$

$=\left\{g h: \begin{array}{l}\text { there exists a geodesic } l \subset \operatorname{cl}\left(\mathscr{C}_{\rho}\right) \cap \operatorname{cl}\left(S_{\rho}\left(0, g^{-1}(0)\right)\right) \\ \text { intersecting } \mathrm{cl}\left(B_{\rho}(0)\right) \text { and } \operatorname{cl}\left(B_{\rho}\left(g^{-1}(0)\right)\right) \text {, and } h \in G_{\rho}\end{array}\right\}$.

\section{Restricted Patterson Measures}

In this section we derive the main results of this paper. First we introduce the concept of the $\rho$-restricted Poincaré series and their associated exponent of convergence $\delta_{\rho}$. We then give the construction of $\rho$-restricted Patterson measures which is followed by an analysis of these measures. We shall see that these measures have no atoms and that they have the properties of being $\delta_{\rho}$-harmonic, $\delta_{\rho}$-subconformal on special sets and even $\delta_{\rho}$-conformal on very special sets. Furthermore, we obtain the results that $G_{\rho}$ is of the $\delta_{\rho}$-divergence type and that the Hausdorff dimension of $L\left(G_{\rho}\right)$ is equal to $\delta_{\rho}$.

We remark that throughout we will repeatedly use the following well-known elementary observation from hyperbolic geometry. The proof is an immediate consequence of the hyperbolic cosine rule (cf. [12, p. 148]).

The Complete Hyperbolic Triangle Inequality Let $0<\alpha_{0}<\pi$ be given and consider an arbitrary triangle in hyperbolic space with side lengths $a, b$ and $c$ such that the angle $\alpha$ formed by the sides of lengths $b$ and $c$ is bounded below by $\alpha_{0}$. Then there is a constant $K$ depending only on $\alpha_{0}$ such that $b+c-K \leq a \leq b+c$. Equivalently, $b+c \asymp_{+} a$, with the constant of comparability depending only on $\alpha_{0}$.

Constructing Restricted Patterson Measures.

Recall that classically one associates with a Kleinian group $G$ its Poincaré series $\mathscr{P}(x, s)$, which is given for $x \in \mathbb{D}^{N+1}$ and $s \geq 0$ by

$$
\mathscr{P}(x, s):=\sum_{g \in G} e^{-s d(x, g(0))} .
$$

The absissa of convergence of this series, which is referred to as the exponent of convergence of $G$ or sometimes also as the Poincaré exponent, will be denoted as usual by $\delta=\delta(G)$. More precisely, we have that

$$
\delta:=\inf \{s \geq 0: \mathscr{P}(x, s) \text { converges }\} .
$$

We remark that $\delta$ clearly does not depend on the particular choice of $x$.

We now modify this classical concept as follows. Let $\rho>\rho_{0}$ be given. For $s \geq 0$ and $x \in \mathbb{D}^{N+1}$, we define the $\rho$-restricted Poincaré series $\mathscr{P}_{\rho}(x, s)$ by

$$
\mathscr{P}_{\rho}(x, s):=\sum_{g \in G_{\rho}} e^{-s d(x, g(0))} .
$$

Let $\delta_{\rho}$ denote the absissa of convergence of this series, that is,

$$
\delta_{\rho}:=\inf \left\{s \geq 0: \mathscr{P}_{\rho}(x, s) \text { converges }\right\} .
$$

Again, we remark that $\delta_{\rho}$ clearly does not depend on the particular choice of $x$. Now, note that it is a priori not clear that $\mathscr{P}_{\rho}\left(x, \delta_{\rho}\right)$ is infinite, or in other words, that $G_{\rho}$ is of $\delta_{\rho}$-divergence type. In order to overcome this difficulty, we employ 'the method of a slowly varying function', which was first used by Patterson to construct orbital measures on limit sets of Fuchsian groups 
([7]). Since our construction requires some modifications of the original construction in [7], and also since the literature seems not to contain a description of this method in all its details, we have included such a description in the appendix and we refer to it for the details. However, the essence of the method is that there exists a function $\phi_{\rho}: G_{\rho} \rightarrow \mathbb{R}^{+}$such that for each $x \in \mathbb{D}^{N+1}$ the absissa of convergence of the modified $\rho$-restricted Poincaré series,

$$
\mathscr{P}_{\rho}^{\prime}(x, s):=\sum_{g \in G_{\rho}}\left(\phi_{\rho}(g) e^{-d(x, g(0))}\right)^{s}
$$

stays to be equal to $\delta_{\rho}$, whereas we have that $\mathscr{P}_{\rho}^{\prime}(x, s)$ diverges for $s=\delta_{\rho}$.

Using this modified $\rho$-restricted Poincaré series, we can now mimic the construction of the classical Patterson measure in order to derive our restricted Patterson measures as follows. For $s>\delta_{\rho}$, let $\mu_{\rho, s}^{x}$ denote the orbital measure given for $E \subset \mathbb{D}^{N+1}$ by

$$
\mu_{\rho, s}^{x}(E):=\frac{1}{\mathscr{P}_{\rho}^{\prime}(0, s)} \sum_{g \in G_{\rho}}\left(\phi_{\rho}(g) e^{-d(x, g(0))}\right)^{s} \mathbf{1}_{g(0)}(E),
$$

where $\mathbf{1}_{g(0)}$ refers to the Dirac point mass of weight one at $g(0)$. Fix a sequence $\left(s_{n}\right)$ such that $\lim _{n \rightarrow \infty} s_{n}=\delta_{\rho}$ and such that $s_{n}>\delta_{\rho}$ for all $n$. By Helly's theorem we have that there exists a subsequence, which for simplicity will also be denoted by $\left(s_{n}\right)$, such that the sequence $\left(\mu_{\rho, s_{n}}^{x}\right)$ converges weakly to some measure $\mu_{\rho}^{x}$ for $n$ tending to infinity. Note that the measure $\mu_{\rho}^{x}$ depends on $x$ as well as on the sequence $\left(s_{n}\right)$. Therefore, for any given $\rho$, throughout we shall fix one such sequence $\left(s_{n}\right)$ for all $x \in \mathbb{D}^{N+1}$ and will assume that it is employed universally in the construction of each $\mu_{\rho}^{x}$ (this is justified by a straight forward adaption of an argument in the construction of the classical Patterson measure (cf. [15] Theorem 3.4.1 and/or Lemma 3.4; see also Proposition 4.5)). Clearly, since $\mathscr{P}_{\rho}^{\prime}(x, s)$ diverges for $s=\delta_{\rho}$, it follows that $\mu_{\rho}^{x}$ is supported on $L\left(G_{\rho}\right)$, and we shall refer to $\mu_{\rho}^{x}$ as the $\rho$-restricted Patterson measure associated with $x$ and $G_{\rho}$. For ease of notation we shall often write $\mu_{\rho, s_{n}}$ instead of $\mu_{\rho, s_{n}}^{0}$, and also $\mu_{\rho}$ instead of $\mu_{\rho}^{0}$.

Non-Atomicity, $\delta_{\rho}$-Divergence Type and Hausdorff Dimension.

A first important property of $\rho$-restricted Patterson measures is that they are non-atomic. For the proof we require the following observation which relates $\delta$ with $\delta_{\rho}$ :

Proposition 3.1 For the critical exponent $\delta$ of $G$ we have that $\delta=\lim _{\rho \rightarrow \infty} \delta_{\rho}$.

Proof Recall from [16] that an element $\xi \in L(G)$ is called a $c$-uniformly radial point of $G$, for some $c>0$, if and only if the geodesic ray $s_{\xi}$ from 0 to $\xi$ is contained in $\bigcup_{g \in G} B_{c}(g(0))$. Let $L_{u r}^{c}(G)$ denote the set of all $c$-uniformly radial points of $G$. By generalizing an argument of Bishop and Jones ([3]), we obtained in [16] that for each $\varepsilon>0$ there exists a constant $c(\varepsilon)$ such that

$$
\operatorname{dim}_{H} L_{u r}^{c(\varepsilon)}(G)>\delta-\varepsilon .
$$

Hence, by definition of the Hausdorff dimension (cf. [17]), it follows that:

$$
\sum_{g \in G_{u r}^{c(\varepsilon)}} \operatorname{diam}\left(\Pi_{0}\left(B_{c(\varepsilon)}(g(0))\right)\right)^{\delta-\varepsilon}=\infty,
$$

where $\operatorname{diam}(\cdot)$ refers to the Euclidean diameter in $S^{N}$, and where we have set

$$
G_{u r}^{c(\varepsilon)}:=\left\{g \in G: s_{\xi} \cap B_{c(\varepsilon)}(g(0)) \neq \emptyset \text { for some } \xi \in L_{u r}^{c(\varepsilon)}(G)\right\} .
$$

Now note that if $\varepsilon>0$ is given, then by choosing $\rho$ sufficiently large we see that $L_{u r}^{c(\varepsilon)}(G) \subset$ $L_{r}\left(G_{\rho}\right)$ as well as $G_{u r}^{c(\varepsilon)} \subset G_{\rho}$. Combining these observations, it follows that:

$$
\mathscr{P}_{\rho}(0, \delta-\varepsilon) \geq \sum_{g \in G_{u r}^{c(\varepsilon)}} e^{-(\delta-\varepsilon) d(0, g(0))} \asymp \sum_{g \in G_{u r}^{c(\varepsilon)}} \operatorname{diam}\left(\Pi_{0}\left(B_{\rho}(g(0))\right)\right)^{\delta-\varepsilon}=\infty,
$$

which implies that $\delta_{\rho} \geq \delta-\varepsilon$. The statement of the lemma now follows, since $\varepsilon$ was chosen to be arbitrary, and since obviously $\delta_{\rho} \leq \delta$. 
We are now in a position to give some preliminary geometric measure estimates for $\mu_{\rho}^{x}$. These estimates represent the main ingredients for proving that $\mu_{\rho}^{x}$ is non-atomic and that $G_{\rho}$ is of $\delta_{\rho}$-divergence type.

Proposition 3.2 For each $x \in \mathbb{D}^{N+1}$ and $\rho$ sufficiently large, the $\rho$-restricted Patterson measure $\mu_{\rho}^{x}$ has the following properties:

(i) If $\xi \in L_{r}\left(G_{\rho}\right)$, then for each element $g_{m}$ in the $\rho$-trace at $\xi$ we have that

$$
\mu_{\rho}^{x}\left(\partial S_{\rho}\left(0, g_{m}(0)\right)\right) \ll e^{-\delta_{\rho} d\left(0, g_{m}(0)\right)} ;
$$

(ii) If $\xi \in L_{p}\left(G_{\rho}\right)$, then $\xi=g(p)$ for some $p \in P$ and $g \in G_{\rho}$, where, without loss of generality, we assume that $g$ is chosen such that $d(0, g(0))=\min \left\{d(0, g h(0)): h \in \Gamma_{p}\right\}$ (here $\Gamma_{p}$ refers to the stabiliser of $p$ in $\left.G\right)$. Then, for every $\varepsilon>0$ there exists $r_{\varepsilon}>0$ such that, for each spherical ball $b(\xi, r) \subset S^{N}$ centred at $\xi$ of radius $0<r<r_{\varepsilon}$, we have that

$$
\mu_{\rho}^{x}(b(\xi, r)) \ll e^{-\left(\delta_{\rho}-\varepsilon\right) d(0, g(0))}\left(r e^{d(0, g(0))}\right)^{2\left(\delta_{\rho}-\varepsilon\right)-k(p)},
$$

where $k(p)$ refers to the rank of the parabolic fixed point $p$.

Proof It is clearly sufficient to prove the assertions for $x=0$. In order to prove $(i)$, note that for each $h \in G_{\rho}$ such that $h(0) \in \widehat{S}_{\rho}\left(0, g_{m}(0)\right)$, the 'complete hyperbolic triangle inequality' gives that $d(0, h(0)) \asymp_{+} d\left(0, g_{m}(0)\right)+d\left(g_{m}(0), h(0)\right)$. Combining this with Corollary $2.6(i)$ and Lemma 4.3 , we obtain, for all $n$, that

$$
\begin{aligned}
& \sum_{\substack{h \in G_{\rho} \\
\in \widehat{S}_{\rho}\left(0, g_{m}(0)\right)}}\left(\phi_{\rho}(h) e^{-d(0, h(0))}\right)^{s_{n}} \\
& \asymp e^{-s_{n} d\left(0, g_{m}(0)\right)} \sum_{\substack{h \in G_{\rho} \\
h(0) \in \hat{S}_{\rho}\left(0, g_{m}(0)\right)}}\left(\phi_{\rho}(h) e^{-d\left(0, g_{m}^{-1} h(0)\right)}\right)^{s_{n}} \\
& \leq e^{-s_{n} d\left(0, g_{m}(0)\right)} \sum_{\substack{k \in G_{\rho} \\
k(0) \in \widehat{S}_{\rho}\left(g_{m}^{-1}(0), 0\right)}}\left(\frac{\phi_{\rho}\left(g_{m} k\right)}{\phi_{\rho}(k)} \phi_{\rho}(k) e^{-d(0, k(0))}\right)^{s_{n}} \\
& \ll e^{-s_{n} d\left(0, g_{m}(0)\right)} \mathscr{P}_{\rho}^{\prime}\left(0, s_{n}\right) .
\end{aligned}
$$

Note that in this estimate the final inequality follows since Lemma 4.3 is applicable, and hence $\phi_{\rho}\left(g_{m} k\right) / \phi_{\rho}(k)$ tends to 1 , for $d(0, k(0))$ tending to infinity. Now, if we divide the inequality so obtained by $\mathscr{P}_{\rho}^{\prime}\left(0, s_{n}\right)$ and let $s_{n}$ tend to $\delta_{\rho}$, the statement in $(i)$ follows.

In order to prove (ii), let $\lambda_{r}$ denote the open hyperbolic lens centred at $g(p)$ of Euclidean radius $r$. That is, $\lambda_{r}$ is the intersection of $\mathbb{D}^{N+1}$ with the open Euclidean ball in $\mathbb{R}^{N+1}$ of radius $r$ which is symmetric about the geodesic ray $s_{g(p)}$ and whose boundary sphere is orthogonal to $S^{N}$. Since $\lambda_{r} \cup \partial \lambda_{r}$ is open in the relative Euclidean topology of $\mathbb{D}^{N+1} \cup S^{N}$, and since $\mu_{\rho, s_{n}}\left(\lambda_{r} \cup \partial \lambda_{r}\right)=\mu_{\rho, s_{n}}\left(\lambda_{r}\right)$ for all $n$, the weak convergence of $\mu_{\rho, s_{n}}$ to $\mu_{\rho}$ gives that

$$
\mu_{\rho}\left(\partial \lambda_{r}\right) \leq \liminf _{n \rightarrow \infty} \mu_{\rho, s_{n}}\left(\lambda_{r}\right) .
$$

Also, for $\rho$ sufficiently large, we have, for all $n$, that

$$
\mu_{\rho, s_{n}}\left(\lambda_{r}\right) \leq \mu_{\rho, s_{n}}\left(\bigcup_{f \in \Gamma_{g(p)}(r)} \widehat{S}_{\rho}(0, f(0))\right)+\mu_{\rho, s_{n}}\left(\bigcup_{f \in \Gamma_{g(p)}(r)} B_{2 \rho}(f(0))\right),
$$

where we have set $\Gamma_{g(p)}(r):=\left\{g e \in G_{\rho}: e \in \Gamma_{p}, \partial \lambda_{m} \cap \partial S_{\rho}(0, g e(0)) \neq \emptyset\right\}$. In order to give estimates from above for the terms on the right-hand side of the latter inequality, first note that, for $f, h \in G_{\rho}$ such that $h(0) \in \widehat{S}_{\rho}(0, f(0))$, the 'complete hyperbolic triangle inequality' implies $d(0, h(0)) \asymp+d(0, f(0))+d(f(0), h(0))$. Using this together with Corollary 2.6 (i), we obtain

$$
\mu_{\rho, s_{n}}\left(\bigcup_{f \in \Gamma_{g(p)}(r)} \widehat{S}_{\rho}(0, f(0))\right)
$$




$$
\begin{aligned}
& \leq \frac{1}{\mathscr{P}_{\rho}^{\prime}\left(0, s_{n}\right)} \sum_{f \in \Gamma_{g(p)}(r)} \sum_{\substack{h \in G_{\rho}: \\
h(0) \in \hat{S}_{\rho}(0, f(0))}}\left(\phi_{\rho}(h) e^{-d(0, h(0))}\right)^{s_{n}} \\
& \asymp \frac{1}{\mathscr{P}_{\rho}^{\prime}\left(0, s_{n}\right)} \sum_{f \in \Gamma_{g(p)}(r)} e^{-s_{n} d(0, f(0))} \sum_{\substack{\left.h \in G_{\rho}: \\
f-h_{h(0) \in \hat{S}_{\rho}(f-1}(0), 0\right)}}\left(\phi_{\rho}(h) e^{-d\left(0, f^{-1} h(0)\right)}\right)^{s_{n}} \\
& \leq \frac{1}{\mathscr{P}_{\rho}^{\prime}\left(0, s_{n}\right)} \sum_{f \in \Gamma_{g(p)}(r)} e^{-s_{n} d(0, f(0))} \sum_{\substack{k \in G_{\rho}: \\
k(0) \in \widehat{S}_{\rho}(f-1(0), 0)}}\left(\phi_{\rho}(f k) e^{-d(0, k(0))}\right)^{s_{n}} .
\end{aligned}
$$

Now note that by Lemma 4.1 we have, for each $f \in \Gamma_{g(p)}(r)$, that

$$
\phi_{\rho}(f k) \leq e^{\varepsilon d_{a}(f k(0), k(0))} \phi_{\rho}(k),
$$

for almost all $k \in G_{\rho}$ with $k(0) \in \widehat{S}_{\rho}\left(f^{-1}(0), 0\right)$, where 'almost all' refers to that there may be finitely many exceptions (depending on $\varepsilon$ ). (Here, we have used $d_{a}(x, y)$ to denote the annular distance between two points $x, y \in \mathbb{D}^{N+1}$, that is $d_{a}(x, y):=|d(0, x)-d(0, y)|$.) Also note that since $k(0) \in \widehat{S}_{\rho}\left(f^{-1}(0), 0\right)$, the 'complete hyperbolic triangle inequality' gives that

$$
\begin{aligned}
d_{a}(f k(0), k(0)) & =d(0, f k(0))-d(0, k(0)) \\
& =d(0, f k(0))-d(f(0), f k(0)) \\
& \asymp_{+} \quad d(0, f(0)) .
\end{aligned}
$$

Therefore, in the final sum in (3) we have that $\phi_{\rho}(f k) \ll e^{\varepsilon d(0, f(0))} \phi_{\rho}(k)$. Using this observation, we continue the estimate in (3) as follows:

$$
\begin{aligned}
\mu_{\rho, s_{n}} & \left(\bigcup_{f \in \Gamma_{g(p)}(r)} \widehat{S}_{\rho}(0, f(0))\right) \\
& \ll \frac{1}{\mathscr{P}_{\rho}^{\prime}\left(0, s_{n}\right)} \sum_{f \in \Gamma_{g(p)}(r)} e^{-\left(s_{n}-\varepsilon\right) d(0, f(0))} \sum_{\substack{k \in G_{\rho}: \\
k(0) \in \widehat{S}_{\rho}(f-1}}\left(\phi_{\rho}(k), 0\right) \\
\quad= & \sum_{f \in \Gamma_{g(p)}(r)} e^{-\left(s_{n}-\varepsilon\right) d(0, f(0))} \mu_{\rho, s_{n}}\left(\widehat{S}_{\rho}\left(f^{-1}(0), 0\right)\right) \ll \sum_{f \in \Gamma_{g(p)}(r)} e^{-\left(s_{n}-\varepsilon\right) d(0, f(0))} .
\end{aligned}
$$

For the second term in (2) we obtain, using Lemma 4.1,

$$
\begin{aligned}
\mu_{\rho, s_{n}}\left(\bigcup_{f \in \Gamma_{g(p)}(r)} B_{2 \rho}(f(0))\right) & \ll \frac{1}{\mathscr{P}_{\rho}^{\prime}\left(0, s_{n}\right)} \sum_{f \in \Gamma_{g(p)}(r)}\left(\phi_{\rho}(f) e^{-d(0, f(0))}\right)^{s_{n}} \\
& \ll \frac{1}{\mathscr{P}_{\rho}^{\prime}\left(0, s_{n}\right)} \sum_{f \in \Gamma_{g(p)}(r)} e^{-\left(s_{n}-\varepsilon\right) d(0, f(0))} .
\end{aligned}
$$

Hence combining these two latter estimates with (1) and (2), it follows that:

$$
\mu_{\rho}\left(\partial \lambda_{r}\right) \ll \sum_{f \in \Gamma_{g(p)}(r)} e^{-\left(\delta_{\rho}-\varepsilon\right) d(0, f(0))} \ll e^{-\left(\delta_{\rho}-\varepsilon\right) d(0, g(0))} \sum_{f \in \Gamma_{g(p)}(r)} e^{-\left(\delta_{\rho}-\varepsilon\right) d(g(0), f(0))},
$$

where the second inequality is obtained immediately from the 'complete hyperbolic triangle inequality'. In order to derive the statement in (ii), we can now proceed exactly as in the proof of the 'global measure formula' in the geometrically finite situation, and we refer to [19] for the details (see also [20] for a completely analogous argument in the context of rational maps).

Theorem 3.3 For each $x \in \mathbb{D}^{N+1}$ and $\rho$ sufficiently large, the following hold:

(i) The measure $\mu_{\rho}^{x}$ is non-atomic;

(ii) $G_{\rho}$ is of $\delta_{\rho}$-divergence type; that is, $\mathscr{P}_{\rho}(x, s)$ diverges for $s=\delta_{\rho}$ (note, that this implies that the use of the slowly varying function $\phi_{\rho}$ in the construction of $\mu_{\rho}^{x}$ is redundant);

(iii) For the Hausdorff dimension of $L\left(G_{\rho}\right)$ we have

$$
\operatorname{dim}_{\mathrm{H}}\left(L\left(G_{\rho}\right)\right)=\delta_{\rho}
$$


Proof In order to prove (i) let $k_{\max }:=\max \{k(p): p \in P\}$, and recall that, by a result of Beardon (cf. [21]), we have that $\delta>k_{\max } / 2$, and that by Proposition $3.1, \delta_{\rho}$ tends to $\delta$. Combining these two facts gives that, for each $\varepsilon>0$ sufficiently small and for all $\rho$ sufficiently large, we have that

$$
\delta_{\rho}-\varepsilon>\frac{k_{\max }}{2} .
$$

Hence with this choice of $\varepsilon$ and $\rho$, Proposition 3.2 (ii) immediately implies that $\mu_{\rho}^{x}$ does not have atoms at bounded parabolic points. On the other hand, the fact that radial limit points are no atoms is clearly a direct consequence of a combination of Lemma 2.4 and Proposition 3.2 (i).

For (ii), assume that $\mathscr{P}_{\rho}(x, s)$ converges for $s=\delta_{\rho}$. Since, by Lemma $2.4, L_{r}\left(G_{\rho}\right)$ is contained in the limsup-set of the family $\left\{\partial S_{\rho}(0, g(0))\right\}_{g \in G_{\rho}}$ and since $e^{-d(0, g(0))} \asymp \operatorname{diam}\left(\partial S_{\rho}(0\right.$, $g(0)))$ for all $g \in G_{\rho}$, the Borel-Cantelli lemma gives that $\mu_{\rho}\left(L_{r}\left(G_{\rho}\right)\right)=0$. By Proposition 2.2(ii) we have that $L\left(G_{\rho}\right)=L_{r}\left(G_{\rho}\right) \cup L_{p}\left(G_{\rho}\right)$. Hence, since $L_{p}\left(G_{\rho}\right)$ is countable and since as we have seen above, $\mu_{\rho}$ has no atoms at parabolic points, it follows that $\mu_{\rho}\left(L\left(G_{\rho}\right)\right)=0$. This clearly contradicts the fact that $\mu_{\rho}$ is supported on $L\left(G_{\rho}\right)$.

Now it remains to prove (iii). First note that, by combining the facts that $\delta_{\rho}$ is the absissa of convergence of $\mathscr{P}_{\rho}(x, s)$, that $e^{-d(0, g(0))} \asymp \operatorname{diam}\left(\partial S_{\rho}(0, g(0))\right)$ for all $g \in G_{\rho}$ and that $L\left(G_{\rho}\right)$ coincides with $L_{r}\left(G_{\rho}\right)$ up to a countable set, we immediately obtain the upper bound $\operatorname{dim}_{\mathrm{H}}\left(L\left(G_{\rho}\right)\right) \leq \delta_{\rho}$. For the lower bound we remark that the fact that $\mu_{\rho}$ is non-atomic implies that the measure estimates in Proposition 3.2 can be improved significantly. That is, knowing that $\mu_{\rho}$ has no atoms allows us to establish for $\mu_{\rho}$ the same type of 'upper global formula' obtained in [19] for the classical Patterson measure in the geometrically finite case. The proof for $\mu_{\rho}$ is exactly the same as in the classical situation and we refer to [19] for the details. More precisely, for each $\xi \in L\left(G_{\rho}\right)$ and $t>0$ we have the following 'upper global estimate':

$$
\mu_{\rho}\left(b\left(\xi, e^{-t}\right)\right) \ll e^{-\delta_{\rho} t} e^{-d\left(\xi_{t}, G_{\rho}(0)\right) \tau\left(\xi_{t}\right)} .
$$

Here $\xi_{t} \in s_{\xi}$ is uniquely determined by $d\left(0, \xi_{t}\right)=t$, and, if $\xi_{t} \in g\left(\mathscr{H}_{p}\right)$ for some $g \in G_{\rho}$ and $p \in P$, then $\tau\left(\xi_{t}\right):=\delta_{\rho}-k(p)$, whereas $\tau\left(\xi_{t}\right):=0$ otherwise.

Now, (4) immediately implies that if $G$ has no parabolic elements, or else if $G$ and $\rho$ are such that $\delta_{\rho} \geq k_{\max }$, then we have, for each $\xi \in L\left(G_{\rho}\right)$,

$$
\limsup _{r \rightarrow 0} \frac{\mu_{\rho}(b(\xi, r))}{r^{\delta_{\rho}}} \ll 1 .
$$

Hence, an application of the 'mass distribution principle for Hausdorff measures' (cf. [22] and/or [23]) gives the lower bound $\delta_{\rho}$ for the Hausdorff dimension in this special situation.

For the remaining cases we employ the following Khintchine-type argument. Let $\kappa>0$ be given, and fix a sequence $\left(t_{n}\right)$ such that $t_{n}>\left(\delta_{\rho} \kappa n+2 \log n\right) /\left(2 \delta_{\rho}-k_{\max }\right)$ for all $n$. By Lemma 4.4 there exists $n_{\varepsilon}^{\prime}$ such that, for all $n \geq n_{\varepsilon}^{\prime}$, we have

$$
\text { card } A_{\rho}(n):=\operatorname{card}\left\{g \in G_{\rho}: n \leq d(0, g(0))<n+1\right\} \ll e^{(1+\kappa) \delta_{\rho} n} .
$$

Combining this and (4), and defining $r_{p, g}:=\operatorname{diam}\left(g\left(\mathscr{H}_{p}\right)\right)$ for $p \in P$ and $g \in G_{\rho}$ such that $d(0, g(0))=\min \left\{d(0, g h(0)): h \in \Gamma_{p}\right\}$, we obtain

$$
\begin{aligned}
\sum_{p \in P} \sum_{n=n_{\kappa}^{\prime}}^{\infty} \sum_{g \in A_{\rho}(n)} \mu_{\rho}\left(b\left(g(p), e^{-t_{n}} r_{p, g}\right)\right) & \ll \sum_{p \in P} \sum_{n=n_{\kappa}^{\prime}}^{\infty} \sum_{g \in A_{\rho}(n)} r_{p, g}^{\delta_{\rho}} e^{-t_{n}\left(2 \delta_{\rho}-k_{\max }\right)} \\
& \ll \sum_{n=n_{\kappa}^{\prime}}^{\infty} e^{\delta_{\rho} \kappa n} e^{-t_{n}\left(2 \delta_{\rho}-k_{\max }\right)} \\
& \ll \sum_{n=1}^{\infty} n^{-2}<\infty .
\end{aligned}
$$

Hence, by applying the Borel-Cantelli lemma, we now have the following. For $\mu_{\rho}$-almost every $\xi \in L\left(G_{\rho}\right)$ there exists $t^{*}=t^{*}(\xi)$ such that, for each $t>t^{*}$, we have that, if $\xi_{t} \in g\left(\mathscr{H}_{p}\right)$ for 
some $g \in G_{\rho}$ and $p \in P$ such that $r_{p, g} \asymp e^{-n}$ for some $n$, then

$$
d\left(\xi_{t}, G_{\rho}(0)\right) \leq \frac{\delta_{\rho} \kappa n+2 \log n}{2 \delta_{\rho}-k_{\max }} .
$$

Note that if $\delta_{\rho} \geq k(p)$ then there is nothing to prove. Hence, we can assume without loss of generality that $\delta_{\rho}<k(p)$. Define $k_{\max }^{*}:=\max \left\{k(q): q \in P, \delta_{\rho}<k(q)\right\}$, and fix a number $\sigma$ such that

$$
\sigma>\frac{\left(\delta_{\rho} \kappa+2(\log n) / n\right)\left(k_{\max }^{*}-\delta_{\rho}\right)}{2 \delta_{\rho}-k_{\max }} .
$$

By combining (4) and (5), the following holds for $\mu_{\rho}$-almost every $\xi$ and for each $t>t^{*}$. Here we can assume without loss of generality that $\xi_{t} \in g\left(\mathscr{H}_{p}\right)$ for some $g \in G_{\rho}$ and $p \in P$ such that $r_{p, g} \asymp e^{-n}$ for some $n$ (note that in this situation we have that $t>n$ ),

$$
\begin{aligned}
\mu_{\rho}\left(b\left(\xi, e^{-t}\right)\right) & \ll e^{-t \delta_{\rho}} e^{-d\left(\xi_{t}, G_{\rho}(0)\right) \tau\left(\xi_{t}\right)} \\
& \ll e^{-t \delta_{\rho}} \exp \left(\frac{\left(k_{\max }^{*}-\delta_{\rho}\right)\left(\delta_{\rho} \kappa n+2 \log n\right)}{2 \delta_{\rho}-k_{\max }}\right) \\
& \ll e^{-t\left(\delta_{\rho}-\sigma\right)} e^{-t \sigma} r_{p, g}^{-\sigma} \leq e^{-t\left(\delta_{\rho}-\sigma\right)} .
\end{aligned}
$$

Since $\kappa$ was chosen to be arbitrary, we have that $\sigma$ can be made arbitrarily small. Hence, it follows that for $\mu_{\rho}$-almost every $\xi$, we have

$$
\liminf _{r \rightarrow 0} \frac{\log \mu_{\rho}(b(\xi, r))}{\log r} \geq \delta_{\rho} .
$$

Now, by employing once more 'the mass distribution principle for Hausdorff measures', the theorem follows.

\section{Harmonicity and Subconformality.}

We end this section by giving two results, which will mark the similarities between the classical and the $\rho$-restricted Patterson measures. We now see first that varying the base point $x$ of a $\rho$-restricted Patterson measure $\mu_{\rho}^{x}$ does not alter its measure class. This will follow from the fact that $\rho$-restricted Patterson measures enjoy the property of being $\delta_{\rho}$-harmonic, meaning that, for arbitrary $x, y \in \mathbb{D}^{N+1}$, the Radon-Nikodym derivative of $\mu_{\rho}^{y}$ with respect to $\mu_{\rho}^{x}$ at some arbitrary $\xi \in L\left(G_{\rho}\right)$, has the property

$$
\frac{d \mu_{\rho}^{y}}{d \mu_{\rho}^{x}}(\xi)=e^{\delta_{\rho}\langle x, y\rangle_{\xi}} .
$$

Here we have used the notation of Helgason for the the signed horospherical distance $\langle x, y\rangle_{\xi}$ between $x$ and $y$ at $\xi$ (cf. [24]). That is, $\langle x, y\rangle_{\xi}$ refers to the hyperbolic distance of the two horospheres based at $\xi$ containing $x$ and $y$, respectively, where $\langle x, y\rangle_{\xi}$ is positive if and only if the horosphere at $\xi$ containing $y$ is contained in the horoball bounded by the horosphere at $\xi$ through $x$.

Lemma 3.4 For each $x \in \mathbb{D}^{N+1}$, the measure $\mu_{\rho}^{x}$ is $\delta_{\rho}$-harmonic.

Proof Let $\xi \in L\left(G_{\rho}\right)$ be fixed, and choose a sequence $\left(\lambda_{m}\right)$ of hyperbolic lenses such that $\xi \in \partial \lambda_{m}$ for all $m$, and such that the Euclidean diameter of $\partial \lambda_{m}$ tends to 0 if $m$ tends to infinity. For a given pair $m, n \in \mathbb{N}$, we have

$$
\begin{aligned}
\frac{\mu_{\rho, s_{n}}^{y}\left(\lambda_{m}\right)}{\mu_{\rho, s_{n}}^{x}\left(\lambda_{m}\right)} & =\frac{\sum_{g \in G_{\rho}: g(0) \in \lambda_{m}} e^{-s_{n} d(y, g(0))}}{\sum_{g \in G_{\rho}: g(0) \in \lambda_{m}} e^{-s_{n} d(x, g(0))}} \\
& =\frac{\sum_{g \in G_{\rho}: g(0) \in \lambda_{m}} e^{s_{n}(d(x, g(0))-d(y, g(0)))} e^{-s_{n} d(x, g(0))}}{\sum_{g \in G_{\rho}: g(0) \in \lambda_{m}} e^{-s_{n} d(x, g(0))}} .
\end{aligned}
$$

Observe that if $z \in \mathbb{D}^{N+1}$ tends to $\xi$, then the difference $d(x, z)-d(y, z)$ tends to $\langle x, y\rangle_{\xi}$. Using this observation and by letting $n$ tend to infinity, the latter estimate yields

$$
\inf _{\eta \in \partial \lambda_{m}} e^{\delta_{\rho}\langle x, y\rangle_{\xi}} \leq \frac{\mu_{\rho}^{y}\left(\partial \lambda_{m}\right)}{\mu_{\rho}^{x}\left(\partial \lambda_{m}\right)} \leq \sup _{\eta \in \partial \lambda_{m}} e^{\delta_{\rho}\langle x, y\rangle_{\xi}} .
$$


This implies that

$$
\lim _{m \rightarrow \infty} \frac{\mu_{\rho}^{y}\left(\partial \lambda_{m}\right)}{\mu_{\rho}^{x}\left(\partial \lambda_{m}\right)}=e^{\delta_{\rho}\langle x, y\rangle_{\xi}}
$$

Since the sequence $\left(\lambda_{m}\right)$ was chosen to be arbitrary and since the expression $e^{\delta_{\rho}\langle x, y\rangle_{\xi}}$ is continuous in $\xi$, the lemma follows.

Finally, we now remark that $\rho$-restricted Patterson measures have the following weaker properties of conformality. For keeping the exposition simple, we have restricted the discussion to the case $x=0$. Also, we remark that for each $g \in G_{\rho}$, we have, by definition of $G_{\rho}^{g}$, that the intersection of $\overline{\partial S_{\rho}(g(0), 0)}$ and $L\left(G_{\rho}^{g}\right)$ is a closed subset of $L\left(G_{\rho}\right)$.

Lemma 3.5 (i) The measure $\mu_{\rho}$ is $\delta_{\rho}$-subconformal on special sets. That is, for each $g \in G_{\rho}$ with $B_{\rho}(g(0)) \cap B_{\rho}(0)=\emptyset$, we have for every $A \subset \overline{\partial S_{\rho}(g(0), 0)}$ measurable,

$$
\mu_{\rho}\left(g^{-1} A\right) \leq \int_{A}\left|\left(g^{-1}\right)^{\prime}(\xi)\right|^{\delta_{\rho}} d \mu_{\rho}(\xi) .
$$

(ii) The measure $\mu_{\rho}$ is $\delta_{\rho}$-conformal on very special sets. That is, for each $g \in G_{\rho}$ with $B_{\rho}(g(0)) \cap B_{\rho}(0)=\emptyset$, we have for every $A \subset L\left(G_{\rho}^{g}\right) \cap \overline{\partial S_{\rho}(g(0), 0)}$ measurable,

$$
\mu_{\rho}\left(g^{-1} A\right)=\int_{A}\left|\left(g^{-1}\right)^{\prime}(\xi)\right|^{\delta_{\rho}} d \mu_{\rho}(\xi) .
$$

Proof Fix $g \in G_{\rho}$ as given in the lemma. For the proof of (i) note that Corollary 2.6(i) implies, for each $n \in \mathbb{N}$ and $A^{\prime} \subset \widehat{S}_{\rho}(g(0), 0)$ measurable,

$$
\begin{aligned}
\mu_{\rho, s_{n}}\left(g^{-1} A^{\prime}\right) & =\frac{1}{\mathscr{P}_{\rho}\left(0, s_{n}\right)} \sum_{h \in G_{\rho}: h(0) \in g^{-1}\left(A^{\prime}\right)} e^{-s_{n} d(0, h(0))} \\
& =\frac{1}{\mathscr{P}_{\rho}\left(0, s_{n}\right)} \sum_{h \in G_{\rho}: g h(0) \in A^{\prime}} e^{-s_{n} d(g(0), g h(0))} \\
& \leq \frac{1}{\mathscr{P}_{\rho}\left(0, s_{n}\right)} \sum_{k \in G_{\rho}: k(0) \in A^{\prime}} e^{-s_{n} d(g(0), k(0))} .
\end{aligned}
$$

Hence, by letting $n$ tend to infinity we derive, for each $A \subset \overline{\partial S_{\rho}(g(0), 0)}$ measurable, that

$$
\mu_{\rho}\left(g^{-1} A\right) \leq \mu_{\rho}^{g(0)}(A) .
$$

Combining this estimate and Lemma 3.4, it follows that:

$$
\mu_{\rho}\left(g^{-1} A\right) \leq \int_{A} e^{\delta_{\rho}\langle 0, g(0)\rangle_{\xi}} d \mu_{\rho}(\xi)=\int_{A}\left|\left(g^{-1}\right)^{\prime}(\xi)\right|^{\delta_{\rho}} d \mu_{\rho}(\xi) .
$$

For proving (ii), the idea is to restrict $g^{-1} * \mu_{\rho, s_{n}}$ and $\mu_{\rho, s_{n}}^{g(0)}$ to some closed subset $V_{g}$ of $\mathbb{D}^{N+1} \cup$ $S^{N+1}$ which is given by some suitable extension of $X_{g}:=L\left(G_{\rho}^{g}\right) \cap \overline{\partial S_{\rho}(g(0), 0)}$ to the interior of hyperbolic space. (Here $g * \nu$ refers to the pull back of a measure $\nu$, that is $g * \nu(E):=\nu(g E)$ for each $E$ measurable.) First note that with each $\xi \in X_{g}$ we can associate a geodesic $l(\xi)$ ending at $\xi$ such that $l(\xi)$ is contained in $\operatorname{cl}\left(\mathscr{C}_{\rho}\right)$ and intersects $\operatorname{cl}\left(B_{\rho}(g(0))\right)$ as well as $\operatorname{cl}\left(B_{\rho}(0)\right)$. Then let $r(\xi)$ denote some geodesic ray towards $\xi$ which starts in $\operatorname{cl}\left(B_{\rho}(0)\right)$ and which is fully contained in $l(\xi)$. Define $Y_{g}$ to be the union of the set of balls $\operatorname{cl}\left(B_{\rho}(h(0))\right)$ for which $h \in G_{\rho}^{g}$ such that $\operatorname{cl}\left(B_{\rho}(h(0))\right)$ intersects $r(\xi)$ for some $\xi \in X_{g}$. Similarly, let $Z_{g}$ denote the union of the set of closed horoballs which are based at the elements of $L_{p}\left(G_{\rho}^{g}\right)$ and which have a non-empty intersection with $r(\xi)$ for some $\xi \in X_{g}$. We now define the extension $V_{g}$ which we already mentioned above by $V_{g}:=X_{g} \cup Y_{g} \cup Z_{g}$. Clearly, by construction we have that $V_{g}$ is closed in $\mathbb{D}^{N+1} \cup S^{N+1}$. Now, consider $\left.g^{-1} * \mu_{\rho, s_{n}}\right|_{V_{g}}$ and $\left.\mu_{\rho, s_{n}}^{g(0)}\right|_{V_{g}}$, the restrictions of $g^{-1} * \mu_{\rho, s_{n}}$ and $\mu_{\rho, s_{n}}^{g(0)}$ to $V_{g}$. A calculation similar to (6), using (ii) of Corollary 2.6 instead of (i), yields that $\left.g^{-1} * \mu_{\rho, s_{n}}\right|_{V_{g}}=\left.\mu_{\rho, s_{n}}^{g(0)}\right|_{V_{g}}$. Recall that in general if a sequence of measures converges weakly to some limit measure then the restrictions of the elements of this sequence to some closed set 
converge weakly to the limit measure restricted to the same closed set. This general observation implies that $\left(\left.\mu_{\rho, s_{n}}^{g(0)}\right|_{V_{g}}\right)$ converges weakly to $\left.\mu_{\rho}^{g(0)}\right|_{V_{g}}$, and also that $\left(\left.g^{-1} * \mu_{\rho, s_{n}}\right|_{V_{g}}\right)$ converges weakly to $\left.g^{-1} * \mu_{\rho}\right|_{V_{g}}$. Since, as we have seen above, the sequences $\left(\left.g^{-1} * \mu_{\rho, s_{n}}\right|_{V_{g}}\right)$ and $\left(\left.\mu_{\rho, s_{n}}^{g(0)}\right|_{V_{g}}\right)$ coincide, it follows that $\left.g^{-1} * \mu_{\rho}\right|_{V_{g}}=\left.\mu_{\rho}^{g(0)}\right|_{V_{g}}$. Hence, as in the proof of (i) above, an application of Lemma 3.4 finishes the proof of (ii).

\section{Appendix}

\section{The Method of a Slowly Varying Function.}

With the notation of Section 3, let the elements of $G_{\rho}$ be ordered such that

$$
G_{\rho}=\left\{g_{0}, g_{1}, g_{2}, \ldots\right\}
$$

where $d\left(0, g_{n}(0)\right) \leq d\left(0, g_{n+1}(0)\right)$ for all $n$. Also, throughout, let $\left(\varepsilon_{n}\right)$ denote some fixed sequence of positive reals which decreases to 0 strictly monotonically. The slowly varying function $\phi_{\rho}$ : $G_{\rho} \mapsto \mathbb{R}^{+}$associated with $\mathscr{P}_{\rho}(x, s)$ and $\left(\varepsilon_{n}\right)$ is then defined by way of induction as follows.

Step 0: Let $\phi_{\rho}\left(g_{0}\right):=1$ and $k_{0}:=0$.

Step 1: Let $t_{0}:=d\left(0, g_{0}(0)\right)(=0)$, and for $i \in\left\{k_{0}+1, \ldots, k_{1}\right\}$ define

$$
\phi_{\rho}\left(g_{i}\right):=e^{\varepsilon_{0}\left(d\left(0, g_{i}(0)\right)-t_{0}\right)}\left(=e^{\varepsilon_{0} d\left(0, g_{i}(0)\right)}\right),
$$

where $k_{1}$ is implicitly determined by

$$
1+\left(\phi_{\rho}\left(g_{k_{1}}\right) e^{-d\left(0, g_{k_{1}}(0)\right)}\right)^{\delta_{\rho}}>\sum_{i=k_{0}+1}^{k_{1}}\left(\phi_{\rho}\left(g_{i}\right) e^{-d\left(0, g_{i}(0)\right)}\right)^{\delta_{\rho}} \geq 1 .
$$

(Note that, the existence of $k_{1}$ follows since $\delta_{\rho}$ is the absissa of convergence of $\mathscr{P}_{\rho}(0, s)$, and hence $\sum_{i \in \mathbb{N}} e^{-\delta_{\rho}\left(1-\varepsilon_{0}\right) d\left(0, g_{i}(0)\right)}$ diverges.)

Step 2: Let $t_{1}:=d\left(0, g_{k_{1}}(0)\right)$, and for $i \in\left\{k_{1}+1, \ldots, k_{2}\right\}$ define

$$
\phi_{\rho}\left(g_{i}\right):=\phi_{\rho}\left(g_{k_{1}}\right) e^{\varepsilon_{1}\left(d\left(0, g_{i}(0)\right)-t_{1}\right)}\left(=e^{\varepsilon_{0}\left(t_{1}-t_{0}\right)} e^{\varepsilon_{1}\left(d\left(0, g_{i}(0)\right)-t_{1}\right)}\right),
$$

where $k_{2}$ is implicitly determined by

$$
1+\left(\phi_{\rho}\left(g_{k_{2}}\right) e^{-d\left(0, g_{k_{2}}(0)\right)}\right)^{\delta_{\rho}}>\sum_{i=k_{1}+1}^{k_{2}}\left(\phi_{\rho}\left(g_{i}\right) e^{-d\left(0, g_{i}(0)\right)}\right)^{\delta_{\rho}} \geq 1 .
$$

(Note that, the existence of $k_{2}$ follows since

$$
\begin{aligned}
\sum_{i=k_{1}+1}^{\infty}\left(\phi_{\rho}\left(g_{k_{1}}\right) e^{\varepsilon_{1}\left(d\left(0, g_{i}(0)\right)-t_{1}\right)} e^{-d\left(0, g_{i}(0)\right)}\right)^{\delta_{\rho}} & =\sum_{i=k_{1}+1}^{\infty} e^{\delta_{\rho} t_{1}\left(\varepsilon_{0}-\varepsilon_{1}\right)} e^{-\delta_{\rho}\left(1-\varepsilon_{1}\right) d\left(0, g_{i}(0)\right)} \\
& \geq \sum_{i=k_{1}+1}^{\infty} e^{-\delta_{\rho}\left(1-\varepsilon_{1}\right) d\left(0, g_{i}(0)\right)}
\end{aligned}
$$

where clearly the final series diverges and hence also the first.)

Step $(m+1)$ : Assume that $k_{m}$ has been obtained in Step $m$.

Let $t_{m}:=d\left(0, g_{k_{m}}(0)\right)$, and for $i \in\left\{k_{m}+1, \ldots, k_{m+1}\right\}$, define

$$
\phi_{\rho}\left(g_{i}\right):=\phi_{\rho}\left(g_{k_{m}}\right) e^{\varepsilon_{m}\left(d\left(0, g_{i}(0)\right)-t_{m}\right)}\left(=e^{\varepsilon_{m}\left(d\left(0, g_{i}(0)\right)-t_{m}\right)} \prod_{j=0}^{m-1} e^{\varepsilon_{j}\left(t_{j+1}-t_{j}\right)}\right),
$$

where $k_{m+1}$ is implicitly determined by

$$
1+\left(\phi_{\rho}\left(g_{k_{m+1}}\right) e^{-d\left(0, g_{k_{m+1}}(0)\right)}\right)^{\delta_{\rho}}>\sum_{i=k_{m}+1}^{k_{m+1}}\left(\phi_{\rho}\left(g_{i}\right) e^{-d\left(0, g_{i}(0)\right)}\right)^{\delta_{\rho}} \geq 1 .
$$


(Note that, the existence of $k_{m+1}$ follows since

$$
\begin{aligned}
\sum_{i=k_{m}+1}^{\infty} & \left(\phi_{\rho}\left(g_{k_{m}}\right) e^{\varepsilon_{m}\left(d\left(0, g_{i}(0)\right)-t_{m}\right)} e^{-d\left(0, g_{i}(0)\right)}\right)^{\delta_{\rho}} \\
& =\sum_{i=k_{m}+1}^{\infty} e^{\delta_{\rho}\left(\varepsilon_{0} t_{1}+\varepsilon_{1}\left(t_{2}-t_{1}\right)+\cdots+\varepsilon_{m-1}\left(t_{m}-t_{m-1}\right)-\varepsilon_{m} t_{m}\right)} e^{-\delta_{\rho}\left(1-\varepsilon_{m}\right) d\left(0, g_{i}(0)\right)} \\
& =\sum_{i=k_{m}+1}^{\infty} e^{\delta_{\rho}\left(t_{1}\left(\varepsilon_{0}-\varepsilon_{1}\right)+t_{2}\left(\varepsilon_{1}-\varepsilon_{2}\right)+\cdots+t_{m}\left(\varepsilon_{m-1}-\varepsilon_{m}\right)\right)} e^{-\delta_{\rho}\left(1-\varepsilon_{m}\right) d\left(0, g_{i}(0)\right)} \\
& \geq \sum_{i=k_{m}+1}^{\infty} e^{-\delta_{\rho}\left(1-\varepsilon_{m}\right) d\left(0, g_{i}(0)\right)}
\end{aligned}
$$

where clearly the final series diverges and hence also the first.)

Before we come to the main result of this appendix, we first give four lemmata in which we collect some technical observations which will turn out to be useful. For the first result recall the notation $d_{a}(z, w)$, which refers to the annular distance between $z$ and $w$ in $\mathbb{D}^{N+1}$, that is, $d_{a}(z, w):=|d(0, z)-d(0, w)|$.

Lemma 4.1 For each $\varepsilon>0$ there exists $n_{\varepsilon} \in \mathbb{N}$ such that, for all $n \geq n_{\varepsilon}$ and all $i \in \mathbb{N}$, we have

$$
\phi_{\rho}\left(g_{n+i}\right) \leq e^{\varepsilon d_{a}\left(g_{n}(0), g_{n+i}(0)\right)} \phi_{\rho}\left(g_{n}\right) .
$$

Proof Fix $0<\varepsilon<1$, and let $m_{0}$ denote the integer which is uniquely determined by $\varepsilon_{m_{0}} \leq$ $\varepsilon<\varepsilon_{m_{0}-1}$. With $\left(k_{m}\right)$ referring to the sequence obtained in the construction of $\phi_{\rho}$ above, define $n_{\varepsilon}:=k_{m_{0}}$. Then for each pair $i, n \in \mathbb{N}$ such that $n \geq n_{\varepsilon}$, there exist $m, m^{\prime} \in \mathbb{N}$ with $m \geq m^{\prime} \geq m_{0}$, such that

$$
\begin{aligned}
& \phi_{\rho}\left(g_{n+i}\right)=e^{\varepsilon_{m}\left(d\left(0, g_{n+i}(0)\right)-t_{m}\right)} \prod_{j=0}^{m-1} e^{\varepsilon_{j}\left(t_{j+1}-t_{j}\right)}, \\
& \phi_{\rho}\left(g_{n}\right)=e^{\varepsilon_{m^{\prime}}\left(d\left(0, g_{n}(0)\right)-t_{m^{\prime}}\right)} \prod_{j=0}^{m^{\prime}-1} e^{\varepsilon_{j}\left(t_{j+1}-t_{j}\right)} .
\end{aligned}
$$

Now, if $m=m^{\prime}$, then we have

$$
\frac{\phi_{\rho}\left(g_{n+i}\right)}{\phi_{\rho}\left(g_{n}\right)}=e^{\varepsilon_{m}\left(d\left(0, g_{n+i}(0)\right)-d\left(0, g_{n}(0)\right)\right)} \leq e^{\varepsilon d_{a}\left(g_{n+i}(0), g_{n}(0)\right)} .
$$

If on the other hand $m>m^{\prime}$, then we have that

$$
\begin{aligned}
\frac{\phi_{\rho}\left(g_{n+i}\right)}{\phi_{\rho}\left(g_{n}\right)} & =e^{\varepsilon_{m}\left(d\left(0, g_{n+i}(0)\right)-t_{m}\right)} e^{\varepsilon_{m^{\prime}}\left(t_{m^{\prime}}-d\left(0, g_{n}(0)\right)\right)} \prod_{j=m^{\prime}}^{m-1} e^{\varepsilon_{j}\left(t_{j+1}-t_{j}\right)} \\
& =e^{\sum_{j=m^{\prime}}^{m-1} \varepsilon_{j}\left(t_{j+1}-t_{j}\right)}+\varepsilon_{m}\left(d\left(0, g_{n+i}(0)\right)-t_{m}\right)+\varepsilon_{m^{\prime}}\left(t_{m^{\prime}}-d\left(0, g_{n}(0)\right)\right)
\end{aligned}
$$

which proves the lemma if we combine with the following estimate for the exponent in the latter expression:

$$
\begin{aligned}
\sum_{j=m^{\prime}}^{m-1} & \varepsilon_{j}\left(t_{j+1}-t_{j}\right)+\varepsilon_{m}\left(d\left(0, g_{n+i}(0)\right)-t_{m}\right)+\varepsilon_{m^{\prime}}\left(t_{m^{\prime}}-d\left(0, g_{n}(0)\right)\right) \\
& \leq \sum_{j=m^{\prime}}^{m-1} \varepsilon_{m^{\prime}}\left(t_{j+1}-t_{j}\right)+\varepsilon_{m^{\prime}}\left(d\left(0, g_{n+i}(0)\right)-t_{m}+t_{m^{\prime}}-d\left(0, g_{n}(0)\right)\right) \\
& =\varepsilon_{m^{\prime}}\left(d\left(0, g_{n+i}(0)\right)-d\left(0, g_{n}(0)\right)\right) \leq \varepsilon d_{a}\left(g_{n+i}(0), g_{n}(0)\right) .
\end{aligned}
$$


The following two lemmas are immediate consequences of the previous lemma. Here $A_{\rho}(n)$ refers to the $n$-annulus of $G_{\rho}$, which is defined, for $n \in \mathbb{N} \cup\{0\}$, by

$$
A_{\rho}(n):=\left\{g \in G_{\rho}: n \leq d(0, g(0))<n+1\right\} .
$$

Also, we let $[\sigma]$ denote the integer part of a non-negative real number $\sigma$.

Lemma 4.2 For every $\varepsilon>0$ and for all $n \geq n_{\varepsilon}$ and $j \in \mathbb{N}$, we have for each $g \in$ $A_{\rho}\left(\left[d\left(0, g_{n}(0)\right)\right]+j\right)$ that $\phi_{\rho}(g) \leq e^{\varepsilon(j+1)} \phi_{\rho}\left(g_{n}\right)$.

Proof By Lemma 4.1 we have that $\phi_{\rho}\left(g_{n+i}\right) \leq e^{\varepsilon d_{a}\left(g_{n+i}(0), g_{n}(0)\right)} \phi_{\rho}\left(g_{n}\right)$ for all $n \geq n_{\varepsilon}$ and $i \in \mathbb{N}$. Hence, if $g \in A_{\rho}\left(\left[d\left(0, g_{n}(0)\right)\right]+j\right)$ for some $j$, then it follows that

$$
\phi_{\rho}(g) \leq e^{\varepsilon d_{a}\left(g(0), g_{n}(0)\right)} \phi_{\rho}\left(g_{n}\right) .
$$

Since in this situation $d_{a}\left(g(0), g_{n}(0)\right) \leq j+1$, the lemma follows.

Lemma 4.3 If $\left(f_{n}\right)$ and $\left(h_{n}\right)$ are two sequences of elements of $G_{\rho}$ such that $d\left(0, f_{n}(0)\right) \asymp_{+}$ $d\left(0, h_{n}(0)\right)$ uniformly for all $n$, and such that

$$
\lim _{n \rightarrow \infty} d\left(0, f_{n}(0)\right)=\lim _{n \rightarrow \infty} d\left(0, h_{n}(0)\right)=\infty,
$$

then it follows that

$$
\lim _{n \rightarrow \infty} \frac{\phi_{\rho}\left(f_{n}\right)}{\phi_{\rho}\left(h_{n}\right)}=1
$$

Proof Let $\varepsilon>0$ be fixed. Using Lemma 4.1 and the assumption that $d_{a}\left(f_{n}(0), h_{n}(0)\right)$ is uniformly bounded, we have for each $n \geq n_{\varepsilon}$ that

$$
\left|\log \left(\phi_{\rho}\left(f_{n}\right)\right)-\log \left(\phi_{\rho}\left(h_{n}\right)\right)\right| \leq \varepsilon d_{a}\left(f_{n}(0), h_{n}(0)\right) \ll \varepsilon .
$$

Since $\varepsilon$ was chosen to be arbitrary, the lemma follows.

Finally, we state a result which is not in the context of the slowly varying function $\phi_{\rho}$. Clearly, this result is an immediate consequence of the fact that $\delta_{\rho}$ is the absissa of convergence of $\mathscr{P}_{\rho}(0, s)$, and we omit its proof.

Lemma 4.4 For each $\kappa>0$ there exists $n_{\kappa}^{\prime} \in \mathbb{N}$ such that, for all $n \geq n_{\kappa}^{\prime}$, we have

$$
\operatorname{card}\left(A_{\rho}(n)\right) \leq e^{\delta_{\rho}(1+\kappa)(n+1)} .
$$

Now, the main result of this appendix is stated in the following proposition:

Proposition 4.5 For each $\rho>0$ and $x \in \mathbb{D}^{N+1}$ we have that $\delta_{\rho}$ is the absissa of convergence of $\mathscr{P}_{\rho}^{\prime}(x, s)$ such that $\mathscr{P}_{\rho}^{\prime}(x, s)$ diverges for $s=\delta_{\rho}$.

Proof Since $\mathscr{P}_{\rho}^{\prime}(0, s) \asymp \mathscr{P}_{\rho}^{\prime}(x, s)$ (where the constants involved depend on $x \in \mathbb{D}^{N+1}$ ), it clearly suffices to prove the assertions for $x=0$.

First note that, by construction of the function $\phi_{\rho}$, we have that

$$
\sum_{n=0}^{\infty}\left(\phi_{\rho}\left(g_{n}\right) e^{-d\left(0, g_{n}(0)\right)}\right)^{\delta_{\rho}} \geq 1+\sum_{m=0}^{\infty} \sum_{i=k_{m}+1}^{k_{m+1}}\left(\phi_{\rho}\left(g_{i}\right) e^{-d\left(0, g_{i}(0)\right)}\right)^{\delta_{\rho}} \geq 1+\sum_{m=0}^{\infty} 1 .
$$

Hence, it follows that $\mathscr{P}_{\rho}^{\prime}(0, s)$ diverges for $s=\delta_{\rho}$.

In order to see that $\mathscr{P}_{\rho}^{\prime}(0, s)$ converges for each $s>\delta_{\rho}$, let $\tau>0$ be given and then fix some $0<\varepsilon<1$ and $\kappa>0$ sufficiently small such that $2 \delta_{\rho}(\varepsilon+\kappa) /(1-\varepsilon)<\tau$. Define

$$
N(\varepsilon, \kappa):=\max \left\{\left[d\left(0, g_{n_{\varepsilon}}(0)\right)\right], n_{\kappa}^{\prime}\right\}
$$

where $n_{\varepsilon}$ and $n_{\kappa}^{\prime}$ are determined by Lemma 4.1 and Lemma 4.4, respectively. The aim is to show that the tail $\Sigma_{\tau}(\varepsilon, \kappa)$ of the sum in $\mathscr{P}_{\rho}^{\prime}\left(0, \delta_{\rho}+\tau\right)$ is finite, where

$$
\Sigma_{\tau}(\varepsilon, \kappa):=\sum_{n=0}^{\infty} \sum_{g \in A_{\rho}(n+N(\varepsilon, \kappa))}\left(\phi_{\rho}(g) e^{-d(0, g(0))}\right)^{\delta_{\rho}+\tau} .
$$


In order to see this we use Lemmas 4.2, 4.4 and the special choice of $\varepsilon$ and $\kappa$, which gives

$$
\begin{aligned}
\Sigma_{\tau}(\varepsilon, \kappa) & \ll \sum_{n \geq N(\varepsilon, \kappa)} e^{\varepsilon(n+1)\left(\delta_{\rho}+\tau\right)} \sum_{g \in A_{\rho}(n)}\left(e^{-d(0, g(0))}\right)^{\delta_{\rho}+\tau} \\
& \leq \sum_{n \geq N(\varepsilon, \kappa)} e^{\varepsilon\left(\delta_{\rho}+\tau\right)(n+1)} e^{-\left(\delta_{\rho}+\tau\right) n} \operatorname{card}\left(A_{\rho}(n)\right) \\
& \ll \sum_{n \geq N(\varepsilon, \kappa)} e^{\varepsilon\left(\delta_{\rho}+\tau\right)(n+1)-\left(\delta_{\rho}+\tau\right)(n+1)+\delta_{\rho}(1+\kappa)(n+1)} \\
& =\sum_{n \geq N_{\varepsilon, \kappa}} e^{-\left((1-\varepsilon) \tau-\delta_{\rho}(\varepsilon+\kappa)\right)(n+1)}<\sum_{n \geq N(\varepsilon, \kappa)} e^{-(n+1) \delta_{\rho}(\varepsilon+\kappa)}<\infty .
\end{aligned}
$$

\section{References}

[1] Sullivan, D.: The density at infinity of a discrete group of hyperbolic motions. IHES Publ. Math., 50, 171-202 (1979)

[2] Sullivan, D.: Discrete conformal groups and measurable dynamics. Bull. Amer. Math. Soc., 6, 57-73 (1982)

[3] Bishop, C. J., Jones, P. W.: Hausdorff dimension and Kleinian groups. Acta Math., 179, 1-39 (1997)

[4] Canary, R. D.: Ends of hyperbolic 3-manifolds. J. Amer. Math. Soc., 6, 1-35 (1993)

[5] Sullivan, D.: Growth of positive harmonic functions and Kleinian group limit sets of planar measure 0 and Hausdorff dimension 2', in Geometry Symposium (Utrecht 1980), Springer, Lect. Notes in Math., 894, $127-144,1981$

[6] Sullivan, D.: A finiteness theorem for cusps. Acta Math., 147, 289-299 (1981)

[7] Patterson, S. J.: The limit set of a Fuchsian group. Acta Math., 136, 241-273 (1976)

[8] Sullivan, D.: Entropy, Hausdorff measures old and new, and limit sets of geometrically finite Kleinian groups. Acta Math., 153, 259-277 (1984)

[9] Denker, M., Urbański, M.: On Sullivan's conformal measures for rational maps of the Riemann sphere. Nonlinearity, 4, 365-384 (1991)

[10] Denker, M., Urbański, M.: On the existence of conformal measures. Transact. Amer. Math. Soc., 328(2), 563-587 (1991)

[11] Urbański, M.: Measures and dimensions in conformal dynamics, preprint, Stony Brook survey article, 1999

[12] Beardon, A. F.: The Geometry of Discrete Groups, Springer Verlag, New York, 1983

[13] Kapovich, M.: Hyperbolic Manifolds and Discrete Group: Lectures on Thurstons Hyperbolization, University of Utah Lecture Notes, 1999

[14] Beardon, A. F., Maskit, B.: Limit points of Kleinian groups and finite sided fundamental polyhedra. Acta Math., 132, 1-12 (1974)

[15] Nicholls, P. J.: The Ergodic Theory of Discrete Groups, Cambridge University Press, Cambridge, 1989

[16] Stratmann, B. O.: The exponent of convergence of Kleinian groups; on a theorem of Bishop and Jones. Mathematica Gottingensis, 1, 1-16 (2001)

[17] Stratmann, B. O.: A note on counting cuspidal excursions. Ann. Acad. Sci. Fenn., 20, 359-372 (1995)

[18] Falconer, K. J.: Fractal Geometry, Mathematical Foundations and Applications, J. Wiley, 1990

[19] Stratmann, B. O., Velani, S.: The Patterson measure for geometrically finite groups with parabolic elements, new and old. Proc. Lond. Math. Soc., 71, 197-220 (1995)

[20] Stratmann, B. O., Urbański, M.: The geometry of conformal measures of parabolic rational maps. Math. Proc. Cambridge Philos. Soc., 128(1), 141-156 (2000)

[21] Beardon, A. F.: The exponent of convergence of Poincaré series. Proc. London Math. Soc., 18, 461-483 (1968)

[22] Falconer, K. J.: Techniques in Fractal Geometry, J. Wiley, 1997

[23] Stratmann, B. O.: Fractal dimensions for Jarník limit sets; the semi-classical approach. Ark. för Mat., 33, 385-403 (1995)

[24] Helgason, S.: Differential Geometry, Lie Groups, and Symmetric Spaces, Acad. Pres, San Diego, 1978 